\title{
Metaanálisis dimensional de la confianza: implicaciones para la comunicación social de la covid-19
}

Dimensional meta-analysis of trust: consequences for social communication of covid-19

Citar como: García Lirios, C. (2020). Metaanálisis dimensional de la confianza: implicaciones para la comunicación social de la covid-19. CITAS, 6(1). https://doi.org/10.15332/24224529.6233

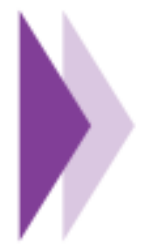

Cruz García Lirios ${ }^{1}$

1 Universidad Autónoma del Estado de México

Correo electrónico: cgarciali@uaemex.mx 


\section{Resumen}

El objetivo de este trabajo es revisar la confianza ciudadana ante la actuación del Gobierno en situaciones de riesgo y contingencia, como la pandemia por la covid-19. Se realizó un estudio documental, metaanalítico y retrospectivo, con una selección de fuentes indexadas en repositorios internacionales. Se consideró el período de 2010 a 2020, aunque el diseño del estudio limitó los resultados al escenario de investigación; se sugirió la extensión del trabajo hacia la relación entre confianza y microfinanzas en el marco del desarrollo local.

Palabras clave: metaanálisis, desarrollo vecinal, riesgo, contingencia, confianza.

\section{Introducción}

La Organización para la Cooperación y el Desarrollo Económico, en su informe para 2020, proyectó un impacto significativo de la pandemia de la enfermedad por coronavirus 2019 (covid-19) en las economías desarrolladas y emergentes (Organización Mundial de la Salud [OMS], 2020). En el mundo, se esperaba un crecimiento del $3 \%$, pero ahora el mejor escenario es del $1 \%$, y el más factible es del $0.5 \%$. En el caso de China se esperaba un crecimiento del 6 $\%$, pero ahora la mejor opción es del $2 \%$, y lo más probable es que no ocurra crecimiento alguno. Japón proyectó un $0.5 \%$, pero su mejor opción será del -0.5\% al -3 \% (Organización Panamericana de la Salud [OPS], 2020). En la misma tendencia negativa, la zona euro aguarda sus opciones de crecimiento: Italia con -2 \%, Alemania y Francia con -1 \%, Gran Bretaña, Estados Unidos y España con $-0.5 \%$.

En este panorama, la confianza entre gobernantes y gobernados es un fenómeno relevante, ya que las decisiones en situaciones de contingencia implican relaciones de confianza entre las partes (Aldana, 2019). En otras palabras, las políticas para contener y mitigar una pandemia implican las percepciones y expectativas de los ciudadanos sobre sus gobernantes, sus estrategias y los resultados esperados. En este sentido, estudios relacionados con la confianza ciudadana respecto a la actuación del Gobierno ante potenciales amenazas a la salud, advierten que la comunicación de riesgos debe ser transparente, vertical y unilateral. Sin embargo, ante la ausencia de resultados favorables o el aumento de la incertidumbre de estos surge la desconfianza y se propaga como una pandemia.

En consecuencia, abordar el fenómeno de la confianza ante una pandemia es fundamental para anticipar escenarios de percepción, comunicación y gestión de potenciales riesgos para la salud, así como la evaluación percibida o esperada de políticas como la contención o mitigación pandémica.

\section{Teoría de las expectativas de confianza}

La estructura de la confianza percibida incluye dimensiones relacionadas con el liderazgo, la tecnología y la ciencia (Bermúdez, 2019). Así, en el caso de la covid-19, la teoría explica la confianza que los ciudadanos depositan en sus líderes políticos respecto a la implementación de la ciencia y la tecnología para solucionar la pandemia.

De esta forma, la confianza es una estructura tripartita, que configura una expectativa acumulada a medida que se acerca un riesgo o se intensifica una crisis (Bucio, 2019). Si la confianza

\section{Abstract}

The objective of this work is to review citizen trust in the government's performance in risk and contingency situations, such as the covid-19 pandemic. A documentary, meta-analytical and retrospective study was carried out, with a selection of sources indexed in international repositories. The period from 2010 to 2020 was considered, although the study design limited the results to the research scenario; the extension of the work to the relationship between trust and microfinance in the framework of local development was suggested.

Keywords: meta-analysis, neighborhood development, risk, contingency, trust.

solo es atribuible a los líderes, entonces se crea un escenario de desesperación adquirida, en el que los ciudadanos aprenden a escuchar a sus líderes, pero sabiendo que no hay solución al problema. En el caso de que los líderes organicen una respuesta integral al problema y tomen sus decisiones con base en la ciencia y la tecnología, la confianza se reconfigura en una expectativa favorable a la identidad global, regional o local.

En el caso de un riesgo esperado e inminente, la confianza aumenta cuando la comunicación del Gobierno se vuelve transparente y técnica. Pero si los resultados se perciben como consecuencia de la negligencia o la ineptitud, entonces la confianza transforma las emociones de incertidumbre, miedo, ansiedad e ira (Bouls, 2020). Durante una contingencia, una vez que los líderes han establecido un curso de acción, la confianza coexiste con las emociones y las percepciones de riesgos, como la inconmensurabilidad, imprevisibilidad e incontrolabilidad de situaciones.

En ese punto, la gestión de riesgos se reduce y la desconfianza aumenta. La confianza en la ciencia y la tecnología emerge en una situación de riesgo inminente y permanece a medida que la comunicación se vuelve transparente, se intensifica y se vuelve técnica. No obstante, en ausencia de metas perceptibles, la confianza se convierte en desesperación o, al menos, en emociones desfavorables.

Sin embargo, la estructura de confianza no se ha establecido en situaciones de máximo riesgo \#como las pandemias\# debido a la frecuencia de su ocurrencia. Así, en un escenario de riesgos para la salud y potencial amenaza a los sectores sociales, la revisión de los estudios es obligatoria.

\section{Estudios de expectativas de confianza}

El sistema político en el que es posible observar las similitudes y diferencias entre grupos a favor y en contra de candidatos presidenciales, a partir de procesos de negociación, mediación, conciliación y arbitraje sobre la gestión y administración de las tecnologías de la información y la comunicación, se conoce como gobernanza (Bustos, 2018; 2019). Este es un fenómeno creciente a medida que se acercan las elecciones locales o federales, y las redes digitales se exacerban como instrumentos para la promoción o disuasión de un candidato.

En el marco de las elecciones presidenciales, la gobernanza se constituye como el sistema de negociación, mediación, conciliación y arbitraje entre los actores involucrados en la gestión y administración de los recursos y servicios públicos (Clark, 2020). En el caso de una contienda electoral anticipada, la gobernabilidad es un fenómeno que refleja preferencias electorales, 
percepciones de consenso e intenciones de votar por partidos, candidatos y sistemas democráticos. En el caso de los efectos de la contienda electoral anticipada en redes digitales, como Facebook, Twitter, YouTube o Instagram, estos se asumen como instrumentos para la promoción de candidatos y plataformas políticas. Es un proselitismo que genera expectativas e intenciones de voto basadas en preferencias electorales, quizás establecidas en medios tradicionales, como la televisión, la radio, los periódicos o el cine, pero cuando se filtran a través de las redes digitales, propician un escenario de debate electoral que \#a efectos del presente estudio\# permite hacer un diagnóstico de la relación que tienen estos determinantes de las elecciones en un futuro próximo.

La psicología social, a través de los modelos de acción razonada y comportamiento planificado, ha influido en la construcción de una psicología de la información. Ambos modelos parten del supuesto de que la conducta está determinada por la relación entre creencias, actitudes, percepciones e intenciones (Carreón, 2018; 2019). Es un proceso que, en el contexto de la información generada en internet, explica las decisiones del consumidor a partir de un procesamiento racional, deliberado, planificado y sistemático.

Los modelos psicosociales se han modificado para ajustar sus relaciones con el procesamiento de la información en internet. Estos son los casos del Modelo de Aceptación de Tecnología, el Modelo de Adopción Comercial y el Modelo de Consumo Electrónico. Estos han incorporado las variables psicosociales de creencias, actitudes, percepciones e intenciones que se propusieron para explicar el comportamiento eficiente y efectivo.

Si bien el acceso a internet es concomitante con el aumento de usuarios de las redes sociales, estas se enfocan en Facebook y Twitter, no solo por la facilidad de uso, sino también por la utilidad de sus protocolos a la hora de difundir información personalizada (García, 2005; 2013; 2019; 2020). Las redes sociales son instancias personalizadas de información, pero el contenido que se difunde en ellas requiere habilidades computacionales, de búsqueda y procesamiento de información, así como capacidades de almacenamiento y difusión. En consecuencia, la brecha digital involucra no solo diferencias entre quienes acceden a los medios digitales y quienes son marginados o excluidos, sino que connota diferencias entre los internautas que buscan información para su entretenimiento y los internautas que procesan información para su conocimiento e innovación. Por ello, los usuarios de internet están adscritos a un sistema de formación académica o profesional, que les obliga a buscar información y procesarla para mostrar un aprendizaje significativo.

La relevancia de las creencias \#entendidas como categorías generales de información\# se extiende a la formación de actitudes definidas como categorías específicas de información, percepciones de riesgo catastrófico o percepciones de utilidad de información asumida como expectativas. Esto permiten anticipar escenarios de incertidumbre, así como las intenciones de usar la internet para procesar la información que se genera (Juárez, 2020). Son las relaciones entre las variables psicosociales las que hacen relevante su inclusión en los modelos psicológicos informacionales, porque explican el procesamiento de la información de eventos lejanos o cercanos a la vida cotidiana de los usuarios de internet. De esta forma, la recepción de información en tiempo real es un factor importante en la planificación de estrategias o estilos de vida que atenúen el impacto de las catástrofes.

La tendencia de los estudios psicológicos informacionales es especificar las variables psicosociales, ya que las creencias son categorizaciones muy generales y no podrían anticipar conductas específicas; aunque las actitudes son categorizaciones más delimitadas, requieren de información percibida para activar decisiones de acción inmediata (Hernández, 2018; 2019). Precisamente porque las intenciones son probabilidades decisivas de llevar a cabo una acción racional, deliberada, planificada y sistemática, predicen la aparición de conductas, pero la información generada en internet conduce a un proceso más emocional que racional. Es por ello por lo que el estudio de intenciones con las dimensión emocional y racional parece ser más pertinente en un escenario impredecible e inconmensurable, como lo serían las contiendas electorales.

Sin embargo, las redes digitales \#como marco de agendas, anuncios, opiniones, preferencias e intenciones\# representan un pequeño porcentaje del electorado que participará en las elecciones (Quiroz, 2019). Esto se debe a que, a diferencia de los medios tradicionales, las redes digitales no solo reproducen información, sino que también generan expectativas en los votantes potenciales. Tal diferencia entre internet, televisión, radio, prensa o cine obliga a reflexionar sobre los estudios de la comunicación de masas centrados en el establecimiento de la agenda, el efecto encuadre y sus consecuencias sobre las intenciones de voto. Es decir que el estudio de las redes digitales supone una diferenciación de sectores, incluso entre los usuarios de Facebook, Twitter, YouTube e Instagram, con respecto a los de otras redes digitales. Por lo tanto, es necesario profundizar en el estudio de las similitudes y diferencias de los internautas de las redes digitales en relación con los potenciales votantes de las elecciones en cuestión.

\section{Especificación de un modelo para el estudio de las expectativas de confianza}

Desde los marcos teórico, conceptual y empírico de la confianza, es posible delinear los ejes, trayectorias y relaciones entre las variables (Rosas, 2019). De esta manera, es posible determinar cómo la confianza hacia las autoridades en cuanto a sus estrategias ante la pandemia por la covid-19 se estructuraría en dimensiones relacionadas con la propensión a las decisiones, pero la aversión a los resultados.

La propensión a las decisiones y la aversión a los resultados configuran un fenómeno ambivalente que prevalece ante los riesgos, contingencias y amenazas esperadas (Limon, 2020). La toma de decisiones, tal como lo establece la teoría de la confianza, involucra una estructura de expectativas basada en costos y beneficios. En el caso de la pandemia, las decisiones de los actores involucrados se establecerán con base en expectativas de altas pérdidas y bajas ganancias. Es decir, los líderes políticos al declarar el confinamiento del pueblo como instrumento de contención o mitigación de la pandemia asumen: 1) el menor número de infecciones y muertes para reducir los efectos económicos, siempre que 2) las instituciones, organizaciones y actores políticos y sociales estén dispuestos a reducir o limitar sus recursos y capacidades.

La estructura de la propensión a las decisiones contrasta con la configuración de la aversión a los resultados (Sánchez, 2019). Es un flujo de expectativas contradictorias, ya que prevalece un consenso de propensión a las decisiones derivado de la identidad universal, la unión nacional o la solidaridad comunitaria, pero contrariamente a los resultados que, para algunos actores, suelen ser mínimos, insuficientes o mejorables.

En este escenario, los líderes buscan preservar su reputación y prestigio (Villegas, 2018; 2019). Mientras tanto, los seguidores cuestionan los resultados, advirtiendo que las decisiones y las consecuencias dependen del grado de innovación del proceso, más que de la optimización de los recursos o, al contrario, si la decisión 
prospectiva es generar nuevas oportunidades. En este sentido, las estrategias para una pandemia son más aceptadas durante una crisis, aunque este apoyo social disminuye a medida que avanza la infección y aumentan las muertes.

\section{Método}

Diseño

Se realizó un estudio documental, retrospectivo y exploratorio.

\section{Muestra}

Se realizó la elección de fuentes indexadas en repositorios internacionales: Academia, Copernicus, Dialnet, Ebsco, Frontiers, Latindex, Redalyc, Scielo, Scopus, WoS, Zenodo y Zotero, considerando el período de publicación de 2010 a 2020. Se realizó la búsqueda incluyendo las palabras clave expectativas y riesgos, y se utilizó la Escala de Expectativa de Confianza, que comprende siete ítems relacionados con las intenciones de los gobernantes con respecto a la protección civil de los gobernados ante eventos de riesgo (tabla 1).

Tabla 1. Dimensión prospectiva de la escala de expectativas de confianza

\begin{tabular}{|l|l|l|l|l|l|}
\hline Repositorio & Año & \multicolumn{1}{|c|}{ Autor } & Muestra & Criterio & \multicolumn{1}{|c|}{ Relación } \\
\hline Academia & 2019 & Villegas & 125 & Formativo & Confianza-Bienestar \\
\hline Copernicus & 2020 & Limón & 100 & Formativo & Confianza-Actitud \\
\hline Dialnet & 2019 & Rosas & 100 & Formativo & Confianza-Percepción \\
\hline Ebsco & 2019 & Hernández & 140 & Formativo & Confianza-Compatibilidad \\
\hline Frontiers & 2019 & Bucio & 130 & Formativo & Confianza-Utilidad \\
\hline Latindex & 2020 & Quiroz & 100 & Formativo & Confianza-Hedonia \\
\hline Redalyc & 2019 & Bermúdez & 130 & Formativo & Confianza-Eudamonia \\
\hline Scielo & 2019 & Juárez & 140 & Formativo & Confianza-Motivación \\
\hline $\begin{array}{l}\text { Scopus } \\
\text { WoS }\end{array}$ & 2019 & Aldana & 110 & Formativo & Confianza-Valores \\
\hline Zenodo & 2019 & Bustos & 100 & Formativo & Confianza-Normas \\
\hline Zotero & 2019 & Sánchez & 120 & Formativo & Confianza-Bienestar \\
\hline
\end{tabular}

Nota: elaborada con base en estudio de datos.

\section{Instrumento}

Se utilizó el inventario de hallazgos sistemáticos de Carreón (2019).

\section{Procedimiento}

Se realizó una búsqueda en www.scholar.google.com considerando las palabras clave: la confianza y los riesgos. Los jueces expertos seleccionaron los estudios teniendo en cuenta las dimensiones teóricas y conceptuales de propensión a la decisión y aversión a los resultados. También se consideraron las cargas factoriales de estas dimensiones en un factor común identificado como expectativas de confianza, perspectiva de consenso o percepción de acuerdo. Los datos se codificaron como: literatura tipo A para los estudios que informaron la preponderancia de ambas dimensiones; y literatura tipo B para los trabajos que incluyeron ambas variables con otras dimensiones como transparencia y rendición de cuentas.

\section{Análisis}

Los datos fueron procesados en las revisiones sistemáticas y mediante el software de metaanálisis versión 3.0. Se consideraron las estadísticas descriptivas, contingentes y proporcionales de probabilidad para toma de decisiones en los umbrales de riesgo permisible.

\section{Resultados}

La tabla 2 muestra el análisis de efectos aleatorios homogéneos de la propensión para toma de decisiones en contingencias, tamaño de la muestra, tamaños del efecto, intervalos de confianza, nivel de significación, varianza, desviación estándar e intervalos de confianza en los umbrales de propensión a la decisión permisible. 
Tabla 2. Análisis del peso de la dimensión prospectiva en la Escala de Expectativas de Confianza

\begin{tabular}{|l|l|l|l|l|l|l|l|}
\hline \multicolumn{1}{|c|}{ Autor } & \multicolumn{1}{|c|}{$\mathbf{N}$} & \multicolumn{1}{|c|}{ TE } & \multicolumn{1}{|c|}{$\boldsymbol{L I}$} & \multicolumn{1}{|c|}{$\boldsymbol{\text { sig. }}$} & \multicolumn{1}{|c|}{$\boldsymbol{V}$} & \multicolumn{1}{|c|}{$\boldsymbol{E}$} \\
\hline Aldana & 110 & .34 & .14 & .25 & .000 & .03 & .12 \\
\hline Bermúdez & 130 & .73 & .17 & .46 & .000 & .03 & .11 \\
\hline Bouls & 130 & .36 & .21 & .57 & .000 & .07 & .09 \\
\hline Bucio & 130 & .20 & .10 & .24 & .000 & .03 & .12 \\
\hline Bustos & 100 & .45 & .22 & .30 & .000 & .03 & .12 \\
\hline Hernández & 140 & .43 & .11 & .62 & .000 & .06 & .36 \\
\hline Juárez & 140 & .30 & .07 & .26 & .000 & .03 & .12 \\
\hline Limón & 100 & .70 & .25 & .40 & .000 & .03 & .11 \\
\hline Quiroz & 100 & .22 & .06 & .39 & .000 & .04 & .16 \\
\hline Rosas & 100 & .64 & .10 & .53 & .000 & .05 & .25 \\
\hline Sánchez & 120 & .56 & .20 & .35 & .000 & .03 & .11 \\
\hline Villegas & 125 & .12 & .05 & .19 & .000 & .03 & .11 \\
\hline
\end{tabular}

Fuente: elaborado con estudio de datos.

Nota: $\mathrm{N}=$ tamaño de muestra; $\mathrm{TE}$ = tamaño de efectos; $\mathrm{LI}$ = límite inferior; $\mathrm{LS}=$ límite superior; sig = nivel de significancia; $\mathrm{V}=$ varianza; $\mathrm{EE}=$ error estándar.

Los datos metanalíticos permitieron estimar los valores de probabilidad proporcional. Estos umbrales de riesgo permisibles sugieren que las expectativas de confianza se encuentran en una zona de tolerancia social. En otras palabras, los estudios que utilizaron la Escala de Propensión a la Decisión parecen mostrar una tenue distancia entre el apoyo ciudadano a las autoridades y su rechazo a las decisiones que tomaron en situaciones de riesgo, más específicamente en la fase de contingencia.

\section{Discusión}

La contribución de este trabajo al estado de la cuestión radica en el establecimiento de umbrales de riesgo tras una revisión sistemática de estudios que utilizaron la Escala de Expectativas de Confianza. Los resultados obtenidos están limitados por los criterios de elección de la investigación que utilizó la escala, el período de revisión y la indexación de la revista. Esto sugiere la extensión de la revisión en otros períodos, repositorios y dimensiones relacionadas con la Escala de Expectativas de Confianza.

En relación con la teoría de las expectativas de confianza \#que propone dos dimensiones, una relacionada con la decisión prospectiva y la otra aludiendo a los resultados esperados\#, el presente trabajo ha establecido un umbral en el que se incluyeron los valores de la revisión sistemática. Las líneas de investigación relativas a la observación de valores metaanalíticos en otros períodos y repositorios permitirán observar el umbral encontrado.

En cuanto a la dimensión prospectiva, que indica el compromiso de altos riesgos para obtener bajos beneficios en un entorno incierto o amenazador, el presente estudio ha establecido un umbral en el que los estudios revisados sistemáticamente son inciertos en una zona permisible o tolerable en relación con las expectativas. digno de confianza. La investigación sobre esta dimensión prospectiva nos permitirá observar el mismo umbral para trabajos que utilizaron la misma escala en diferentes contextos.

En cuanto a la dimensión aversiva de la Escala de Expectativas de Confianza, que resalta la disidencia entre las partes involucradas, este trabajo advierte que la dimensión prospectiva podría no estar relacionada. Esto es así porque la comunicación de riesgos no es un proceso continuo, sino emergente y, por lo tanto, disruptivo. Los estudios que corroboran este supuesto permitirán establecer un umbral percibido como tolerable para quienes esperan decisiones $\mathrm{y}$ acciones de sus autoridades.

\section{Conclusión}

El objetivo del presente trabajo fue establecer umbrales de expectativas de confianza ante eventos de riesgo. En el caso de la dimensión prospectiva, el presente estudio estableció el umbral considerando una revisión de 2010 a 2020, indexación y carga factorial. Estos resultados están limitados por estas restricciones y sugieren una extensión a otro período de tiempo y tipo de repositorio. En relación con el marco teórico, el umbral establecido sugiere un riesgo admisible, aunque probablemente no estaría asociado con el umbral de la dimensión aversiva.

\section{Referencias}

Aldana, W. (2019). Especificación de un modelo para el estudio de las redes colaborativas en una universidad productora del conocimiento. Civilizar. 18(35), 91-102. https://doi.org/10.225 18/usergioa/jour/ccsh/2018.2/a07 
Bermúdez, G. (2019). Validez meta analítica del inventario de emprendimiento social: un estudio del tamaño de los efectos aleatorios. Revista Mundial de Gestión. 19(10), 15-19.

Bouls, B. (2020). Especificación de un modelo de emprendimiento: del oportunismo a la innovación. Revista Internacional de Investigación Avanzada, (3), 20-24.

Bucio, C. (2019). Estructura factorial exploratoria del emprendimiento difícil en una localidad del centro de México. Integración Académica en Psicología, (20), 111-127.

Bustos, J. M. (2019). Efectos bivariados aleatorios de metaanálisis de la gobernanza en la gestión de recursos comunes. Revista Social Science Rsearch, (9), 1-9.

Bustos, J. M. (2018). Contrastación de un modelo de decisión prospectiva e implicaciones para una gobernanza universitaria de la sustentabilidad. Revista Trabajo Social. 23(81), 1-10.

Carreón, J. (2018). Modelamiento del emprendimiento internauta en una universidad pública del centro de México mediante el análisis factorial exploratorio. Divulgación de la Investigación. 16(1), 1-16

Carreón, J. (2019). Modelo de los determinantes del capital humano. Revista Internacional de Avances en Ciencias Sociales y Humanidades, 7(8), 1-5.

Clark, S. (2020). Especificación de un modelo de capital intelectual. Revista internacional de Estudios Económicos y de Gestión. 10(3), 1-3.

García, C. (2005). El modelo de decisión prospectiva. REME, 1(1), $1-10$.

García, C. (2013). La red de conocimiento en una universidad con sistema de prácticas profesionales y servicio social tecnológicoadministrativo. Fundamentos en Humanidades. 14(1), 135-157.

García, C. (2019). Agenda de gestión del conocimiento. Akademia. 18(1), 1-10

García, C. (2020). Especificación de un modelo para el estudio del emprendimiento de servicios públicos. Revista mundial de ciencias sociales humanas. 20(1), 35-38.

Hernández, J. (2018). Modelo de cibercultura organizacional en la innovación del conocimiento. Visión Gerencial. 18(2), 235-253.

Hernández, J. (2019). Decisión prospectiva optimizadora e innovadora. Eureka. 16(2), 127-143.

Juárez, M. (2020). Especificación de un modelo para el estudio de la asistencia cooperativa. Revista global de arqueología y antropología. 11(2), 50-54.

Limon, G. A. (2020). Especificación de un modelo de información sobre el coronavirus covid-19. Revista Internacional de Estudios de Ciencias Sociales, (6), 1-5.

Organización Mundial de la Salud (OMS). (2020). Impacto de la crisis financiera y económica mundial en la salud. OMS. https://www.who.int/mediacentre/news/statements/2009 /financial_crisis_20090401/es/

Organización Panamericana de la Salud (OPS). (2020) La salud y el nuevo orden económico internacional. OPS. https://iris.paho .org/handle/10665.2/6469

Quiroz, C. Y. (2019). Especificación de un modelo de gestión del conocimiento cultural. Revista de búsqueda avanzada global de ciencias agrícolas, 8(10), 1-4.

Rosas, F. J. (2019). Contrastación de un modelo de decisión prospectiva de la sustentabilidad. Invurnus. 14(2), 9-19.

Sánchez, A. (2019). Confiabilidad y validez de un instrumento que mide la gestión del conocimiento. Espacios en Blanco. 30, 9-22.
Villegas, E. (2018). Establecimiento de una política de ciencia y tecnología para la incubación de microempresas innovadoras del conocimiento. Inclusiones, 19-26.

Villegas, E. (2019). Especificación de un modelo para el estudio de la percepción del conocimiento. Open Journal Political Science, (1), 1-6.

cc) (1) 